\author{
Digitai Speckle Fattem Irterferometry (DSPY): \\ A fast procedure to detect and measure vibration mode shapes \\ Pierre Slangen, Léon Berwart, Christophe de Veuster, Jean-Claude Golinval*, Yves Lion \\ HOLOLAB, Laboratoire de Physique Générale, Université de Liège \\ Bât. B5, Sart-Tilman, B-4000, Liège 1, Belgium \\ * L.T.A.S., Aerospace Laboratory, Université de Liège, Bât C3, Val Benoît, B-4000 Liège, Belgium
}

\begin{abstract}
Digital Speckle Pattern Interferometry (DSPI) is a non destructive testing optical method allowing the vizualization of the defects or the deformations of an object submitted to static deformation or to vibration. This method can be applied to a lot of cases within a range of displacements between tens of nanometers and tens of micrometers. DSPI can be applied to detect the natural frequencies and to vizualize the mode shapes of a vibrating object. It is very convenient to study small and weak objects because no contact is required comparing to classical modal analysis using accelerometers. DSPI was successfully applied to study a cantilever aluminium plate $(5 \mathrm{~cm} \times 10 \mathrm{~cm} \times 1 \mathrm{~mm})$. The experimental iso-displacement fringe maps are compared to computational results using a finite element method.
\end{abstract}

\title{
1. PRINCIPLE
}

Illuminating an optically rough object with coherent light yields to a grainy aspect of the surface: the speckle. The size of the speckle depends on the optical system used to form the image onto a sensitive medium (human retina, CCD chip,...). When using an optical system with a focal length $\mathbf{f}$ and an aperture diameter $a$, the size $d_{s}$ of the speckle at the wavelength $\lambda$ is given by:

$$
\mathrm{d}_{\mathrm{s}} \approx \frac{\lambda \mathrm{f}}{\mathrm{a}}
$$

\subsection{Speckle interferometry}

When the speckle pattern interferes with a reference beam as in holographic interferometry, the resulting interferograni is called a specklegram. The reference beam can be an expanded laser beam or an other speckle pattern generated by a reference surface ${ }^{1}$.

The sensitivity to the different components of the displacement is given by the geometry of the interferometer. Figure 1 shows an "out-of-plane" sensitive interferometer (OPI) and figure 2 shows the "in-plane" sensitive set-up (IPI) ${ }^{2}$. With these different set-ups it is then possible to transcode the displacement $\mathbf{d}\left(\mathrm{d}_{\mathrm{x}}, \mathrm{d}_{\mathrm{y}}, \mathrm{d}_{\mathrm{z}}\right)$ of the object to a phase change $\Delta \phi$.

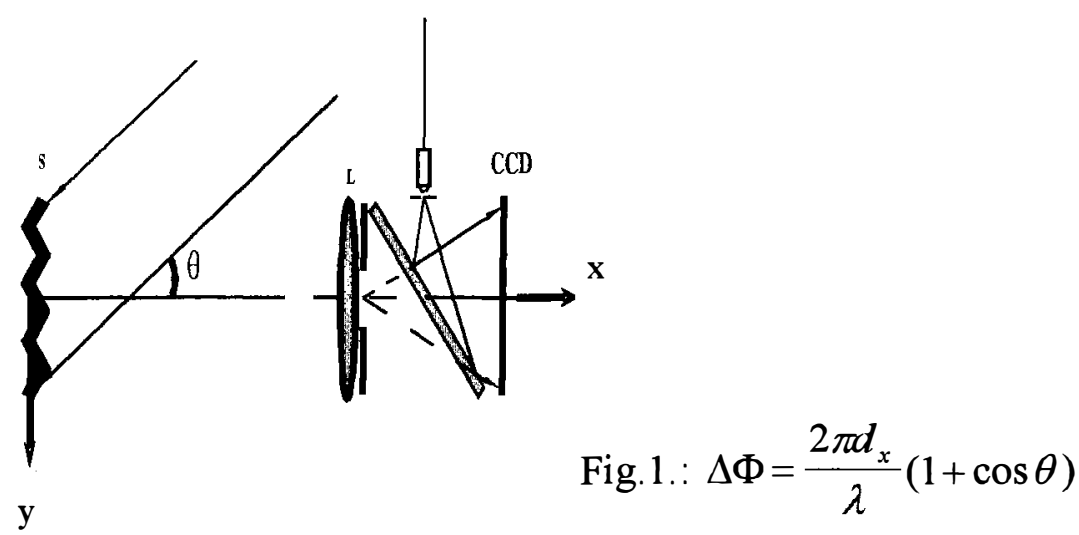




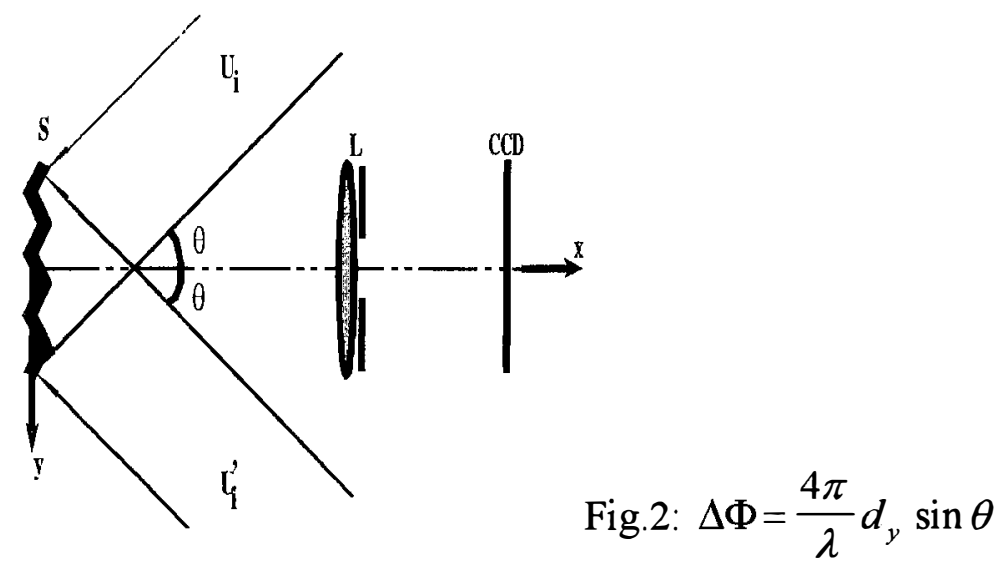

\section{RECORDING OF THE SPECKLEGRAM}

In ESPI (Electronic Speckle Pattern Interferometry), the images are recorded by an electronic camera and processed by analogic filters whereas in DSPI the images are recorded with a CCD camera and digitally processed. Using an appropriate software, the comparison of the images before and after deformation can lead to the map of fringes relative to the displacement in the sensitivity direction. The specklegrams can be processed in the following manners ${ }^{1}$ :

\subsection{Addition of the specklegrams}

In this case, the quality of the fringes is poor because the signal to noise ratio is low. It is mainly due to the superposition of the noise present in the two different specklegrams.

This technique is mainly used when working with a pulsed laser and recording the specklegrams during one snap-shot frame of the CCD.

\subsection{Subtraction of the specklegrams}

In comparison with addition, the quality of the fringes increases. If we assume that the noise present in each specklegram is constant, the $\mathrm{S} / \mathrm{N}$ ratio increases when subtraction occurs. When displayed, the fringes are brighter and the contrast is also better.

This technique is mainly applied to vizualize static deformations but it is also possible to use it to subtract two specklegrams generated with a pulsed laser at two different times e.g. when a dynamic deformation is frozen by the laser pulse.

\subsubsection{Time-averaged specklegrams}

The time-averaged measurement is an extension of the addition method. When the object is vibrating at a natural frequency much higher than the CCD frame refreshing time, the resulting image is the superposition of the different positions of the vibration. It is called a time-averaged specklegram and the nodes of vibration correspond to the brightest fringes $3,4,5,6,7,8$.

This is due to the modulation of the intensity by the function $\mathrm{J}_{0}{ }^{2}$ (zero order Bessel function) resulting from the integration of the vibration mode along a frame time of the CCD:

$$
\frac{1}{\mathrm{~T}} \int_{0}^{\mathrm{T}} \exp (\mathrm{i} \Delta \Phi) \mathrm{dt}
$$


For a single time-averaged specklegram, the contrast is really poor. To enhance the fringes visibility it is worthy to subtract from this first image the steady state specklegram or an other time-averaged specklegram. However the results given by these different methods are still noisy. It is possible to increase the accuracy of the results when applying the phase shifting to the DSPI. Contrast enhancement can also be achieved using speckle averaging in the video frame store ${ }^{9}$. A different technique based on the statistical properties of the dynamic speckle is also used for vibration analysis ${ }^{10}$.

\section{THE PHASE SHIFTING}

The determination of the phase at every points of the object before and after deformation can be achieved by the phase shifting. Knowing the geometry of the interferometer leads automatically to the value of the displacement, in module and direction ${ }^{10}$.

The intensity reaching the $\mathrm{CCD}$ is given by:

$$
I(x, y)=I_{B}(x, y)+I_{M}(x, y) \cos \varphi(x, y)
$$

where $I_{B}$ is the background intensity, $I_{M}$ the modulation intensity, and $\varphi$ the phase. Equation (5) involves three unknowns; $I_{B}, I_{m}$, and $\varphi$. The system can be determined by introducing phase shifts $\alpha_{i}$ as followed:

$$
I_{i}(x, y)=I_{B}(x, y)+I_{M}(x, y) \cos \left[\varphi(x, y)+\alpha_{i}\right]
$$

To solve the resulting system, at least three different intensities must be measured in each pixel of the CCD camera.

Phase shifts have to be known with a three buckets algorithm or can be checked and computed with a four buckets algorithm. Different algorithms using more phase shifts also exist ${ }^{12}$. Simple methods using a single $\pi$ shift between two time-averaged specklegrams have also been developed ${ }^{4}$. Max-min scanning can also produce good results for image quality enhancement but needs a lot of images ${ }^{13}$.

\subsection{Accuracy}

The accuracy of the phase shifting method depends on the accuracy of the experimental set-up. In our case, the phase shift is produced by moving a mirror mounted on a piezoelectric transducer in the reference arm. It is also possible to use polarization techniques or to bend an optical fibre to produce it ${ }^{14,15,16}$.

An accurate calibration of the piezoelectric transducer was done using a Michelson interferometer in the reference path.

The increments of phase are computer-controled and generated with the DAC. We also developed different softwares for the automatic analysis of piezoelectric transducer calibration and also to measure the drift in time of the set-up.

The result is shown in fig. 3 for the calibration. The linearity is really good as well as the reproducibility (about $\lambda / 10$ ). The drift is about $\lambda / 12$ in 100 seconds. 


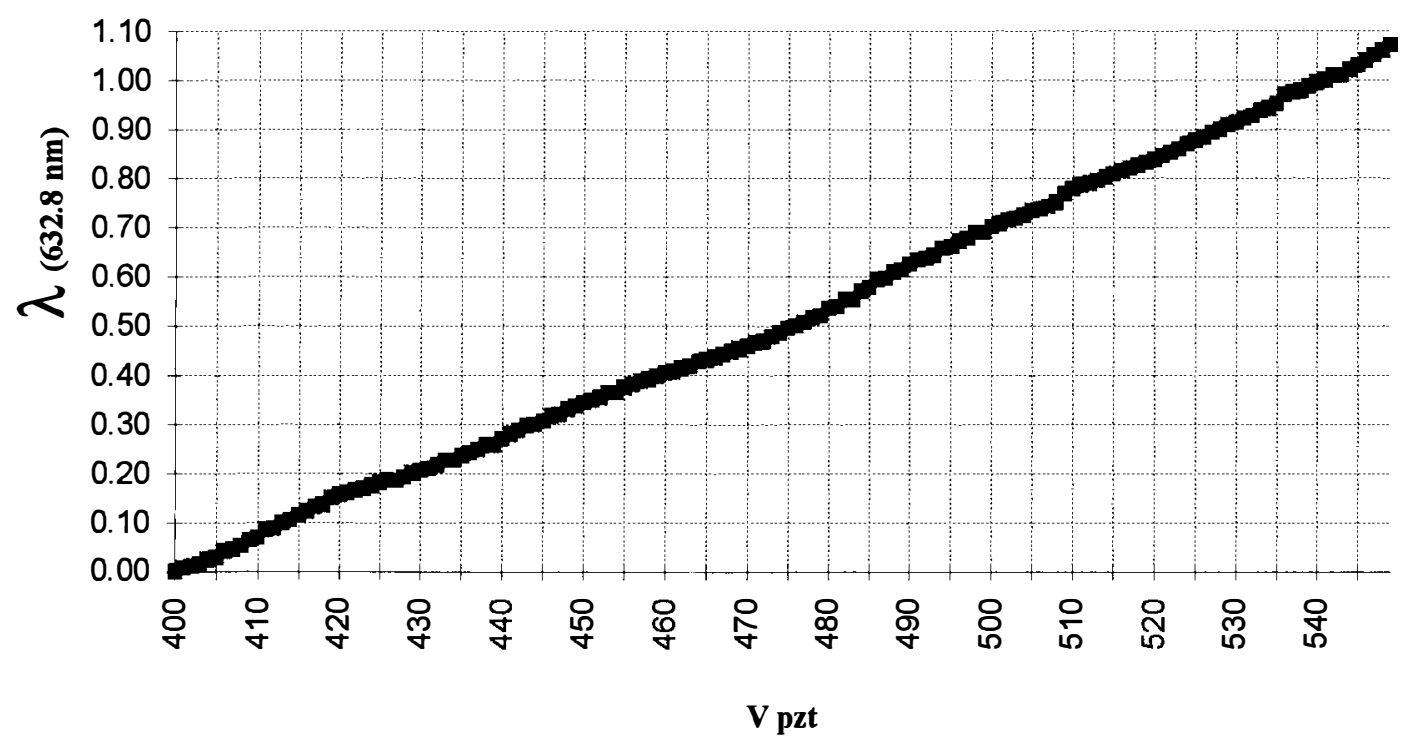

Fig.3.: Calibration of the piezoelectric transducer (PZT)

\subsection{Phase decorrelation}

If the environment of the interferometric set-up is not stable (air flow, temperature gradient,...), the phase can drift with time. So it is obvious that all the measurements have to be made within a very short time.

When measuring an out-of-plane displacement, it has to be taken into account that the speckle generated on the surface is also moving "in-plane". If this in-plane displacement is more important than the speckle size, the decorrelation of the phase appears at the CCD chip (Fig.4.). This is a typical case of the limitation of the DSPI technique to large out-of-plane displacement.

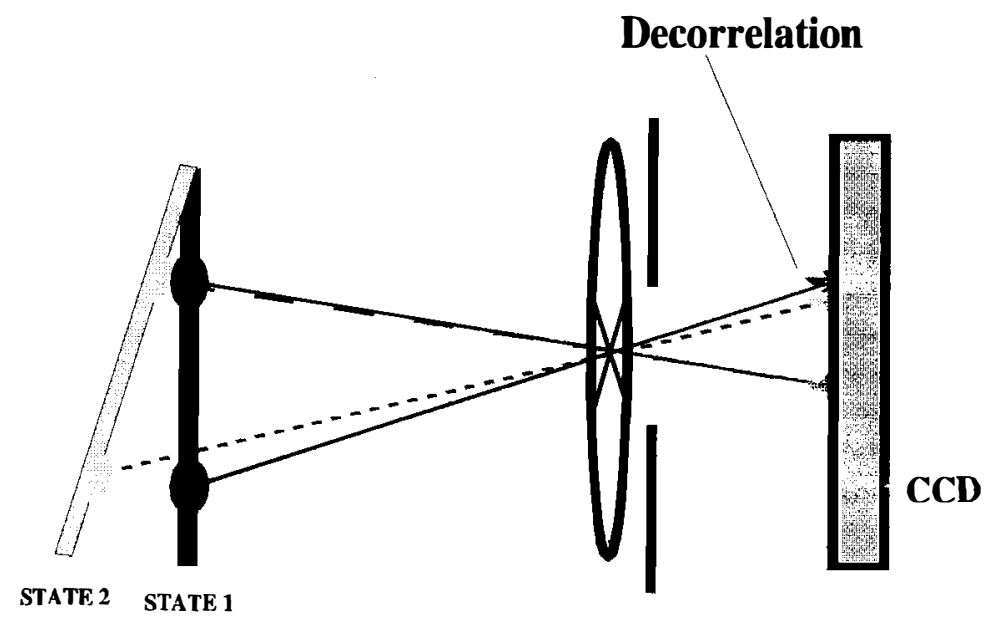

Fig.4.: Decorrelation of the speckle. 


\section{EXPERIMENTAL RESULTS}

The DSPI was applied to detect and vizualize the mode shapes of an aluminium cantilever plate $(5 \mathrm{~cm} \times 7.44 \mathrm{~cm} \times$ $1 \mathrm{~mm})$. The set-up is shown in fig.5. The laser source is a continuous He-Ne laser $(15 \mathrm{~mW}$ power). The piezoelectric transducer (PZT) is computer driven by a Digital/Analogue Converter (DAC) and can generate the required phase shift. The interferometer is out-of-plane sensitive.

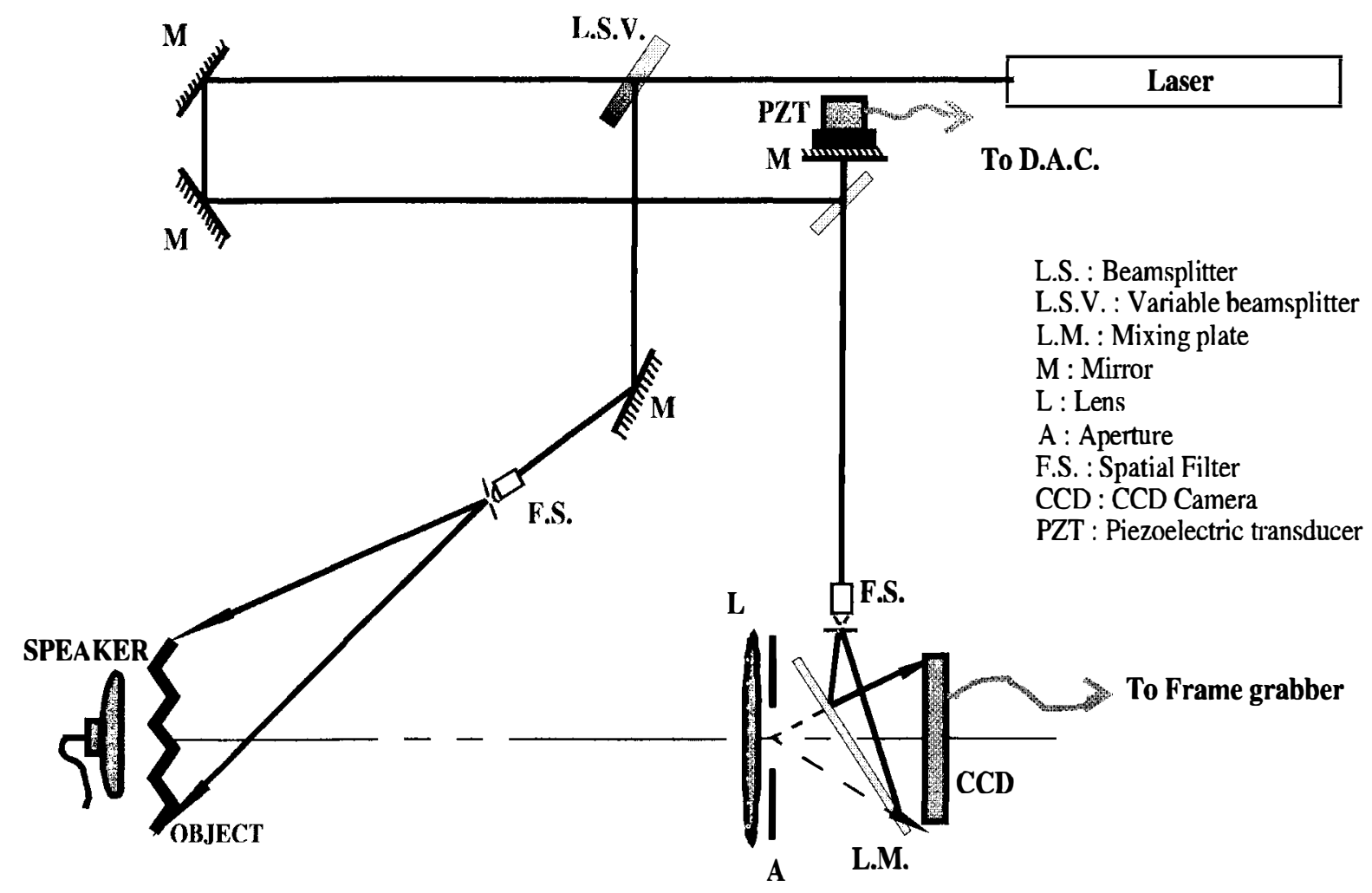

Fig.5.: Phase shifting out-of-plane sensitive interferometer

\subsection{Detection of the natural frequencies}

The cantilever plate is clamped in a mandrel fixed to the optical table: the free edge is $7.44 \mathrm{~cm}$ long (this gives the boundary conditions for finite element analysis). The plate is acoustically excited using a speaker driven by a sinusoidal wave generator. The time-averaged specklegram is displayed in real-time on a TV monitor. During the frequency scanning, the plate vibrates through different vibration modes. These modes are detected by the operator when the fringes are freezing (stationary resonance). Then it is possible to get a more accurate fringes map using the phase shift device. The comparison is done between the steady state and the time-averaged, phase-coded, specklegrams.

The accuracy of the natural frequency is given by the accuracy of the wave generator but also by the skill of the user. This is due to the fact that the fringe contrast is poor while scanning. This should be avoided using a frame grabber that permits the frame speed subtraction. So to enhance the accuracy of the frequency, it is better to first make a fast scan to detect the mode and then to scan nearby the resonance. This method really allows to detect the establishment of the resonance as shown in fig. 6. 


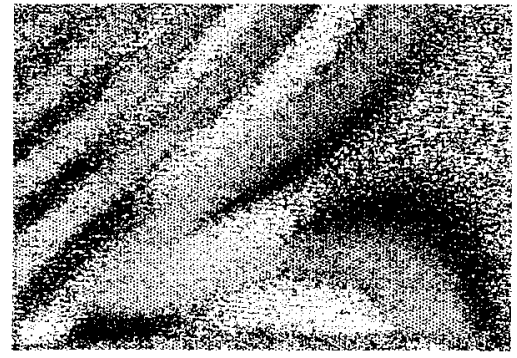

$478 \mathrm{~Hz}$

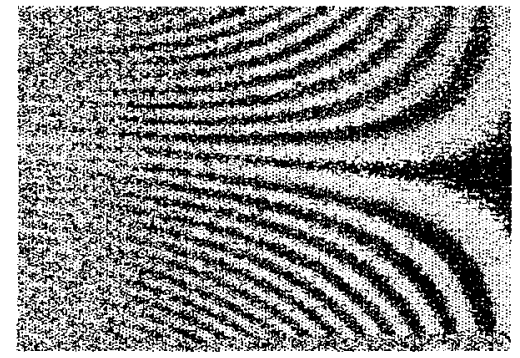

$473 \mathrm{~Hz}$

Fig.6.: Mode tracking: First torsion mode $(473 \mathrm{~Hz})$; clamping on the right side.

Fig.7. shows the result for the cantilever plate vibrating at $3510 \mathrm{~Hz}$.

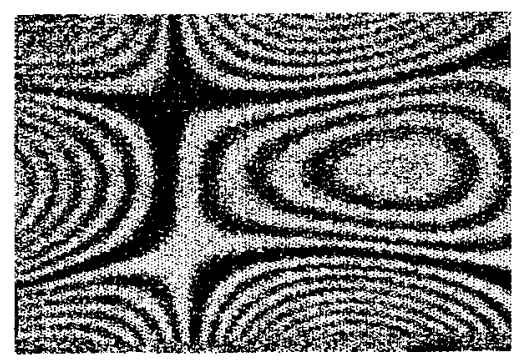

$3510 \mathrm{~Hz}$

Fig.7.: Vibration mode shapes for the cantilever plate: $3510 \mathrm{~Hz}$

\section{FINITE ELEMENT ANALYSIS}

The example considered in this paper is a thin aluminium cantilever plate with overall dimensions of $74.4 \times 50 \times 1 \mathrm{~mm}$ and with material properties of $\mathrm{E}=6.725 .10^{10} \mathrm{~N} / \mathrm{m}^{2}$ and $\rho=2640 \mathrm{~kg} / \mathrm{m}^{3}$.

The finite element (FE) model shown in figure 8 was created with the SAMCEF package ${ }^{17}$.

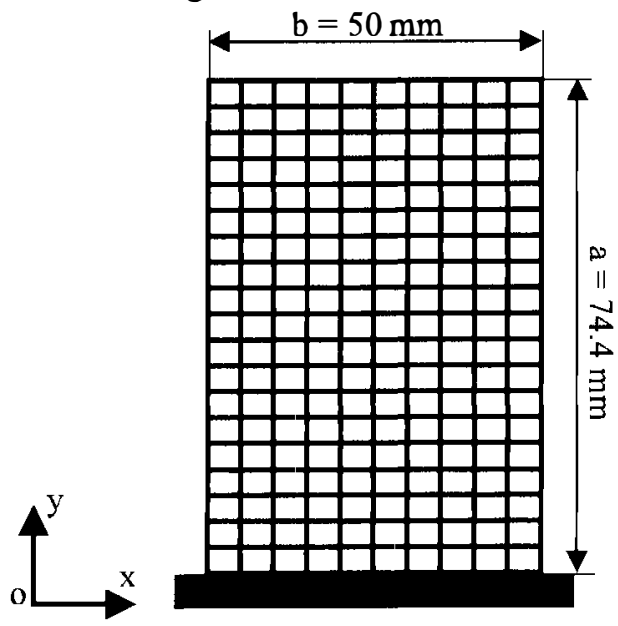

Fig.8: Finite element model of the cantilever plate 
The plate was modelled using 200 four-noded quadratic elements, resulting in a total of 1265 degrees of freedom (DOFs). The degree of correlation between the FE and the experimental models was first investigated by comparison of the first two natural frequencies. Thus the modulus of elasticity was updated on the basis of the following formula for natural frequencies of a clamped plate ${ }^{18}$.

$f_{i j}=\frac{\lambda_{i j}^{2}}{2 \pi a^{2}} \sqrt{\frac{E h^{3}}{12 \gamma\left(1-v^{2}\right)}} ; i=1,2,3 \ldots ; j=1,2,3 \ldots$

where

$\mathrm{i}=$ number of half-waves in mode shape along horizontal y-axis,

$\mathrm{j}=$ number of half-waves in mode shape along horizontal $\mathrm{x}$-axis,

$\lambda_{\mathrm{ij}}=$ dimensionless frequency parameter function of the boundary conditions applied at the edges of the plate,

$\mathrm{a}=$ plate length ,

$\mathrm{b}=$ plate width,

$\mathrm{E}=$ modulus of elasticity,

$\mathbf{h}=$ thickness of plate,

$\gamma=$ mass per unit area of plate,

$v=$ Poisson's ratio.

As the plate is not connected to the clamping device in a perfectly rigid fashion, it was found that the release of the rutational DOFs around $x$-axis allowed a significant improvement of the correlation between the first ten experimental and calculated natural frequencies.

\section{CONCLUSION}

Digital Speckle Pattern Interferometry shows its capability to accurately measure vibration mode shapes of plate or shell siructures. We have shown that it was also possible to detect the natural frequencies with a good accuracy. The comparison with the FE results are in good agreement providing small release for the rotational DOFs to approach the behaviour of the clamping (Fig.9).

As a non intrusive measurement technique, it provides some valuable insights for future industrial applications such as for example the experimental modal analysis of blades.

\section{IMPROVEMENTS}

In case of coupling between modes, a technique permitting the mode separation has been done by Ganesan et al. ${ }^{19}$.

Improvement of the existing set-up can be reach by using optical fibres and a laser diode to get a more compact and easy-to-carry system. Moreover, this can lead to a set-up able to switch between the in-plane and the out-of-plane interferometers and the possibility to analyse the $3-\mathrm{D}$ vibration ${ }^{20}$.

Future research will be focused on the use of DSPI results for structural model updating. 

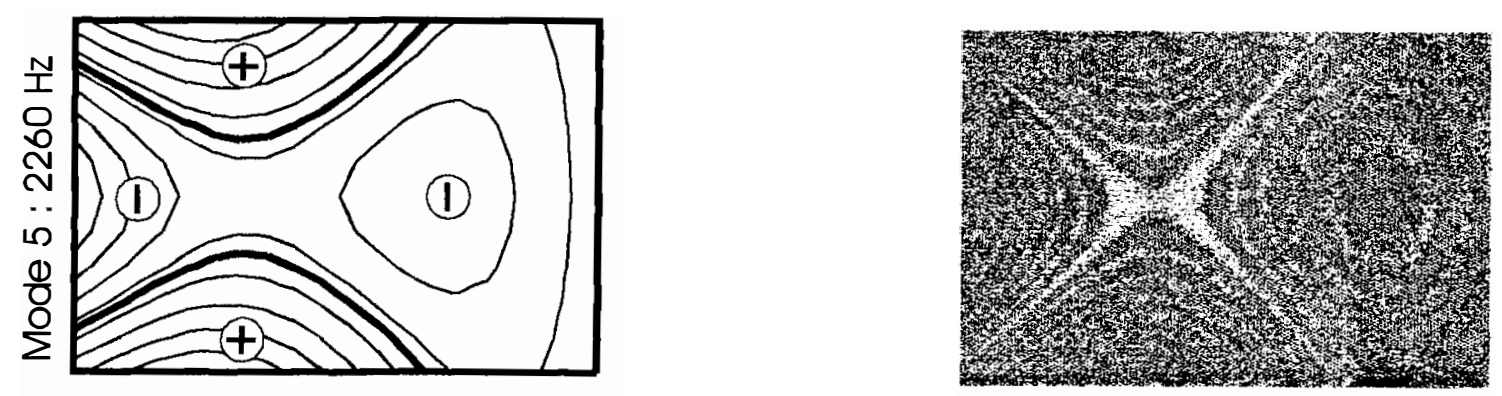

$\frac{2}{2}$
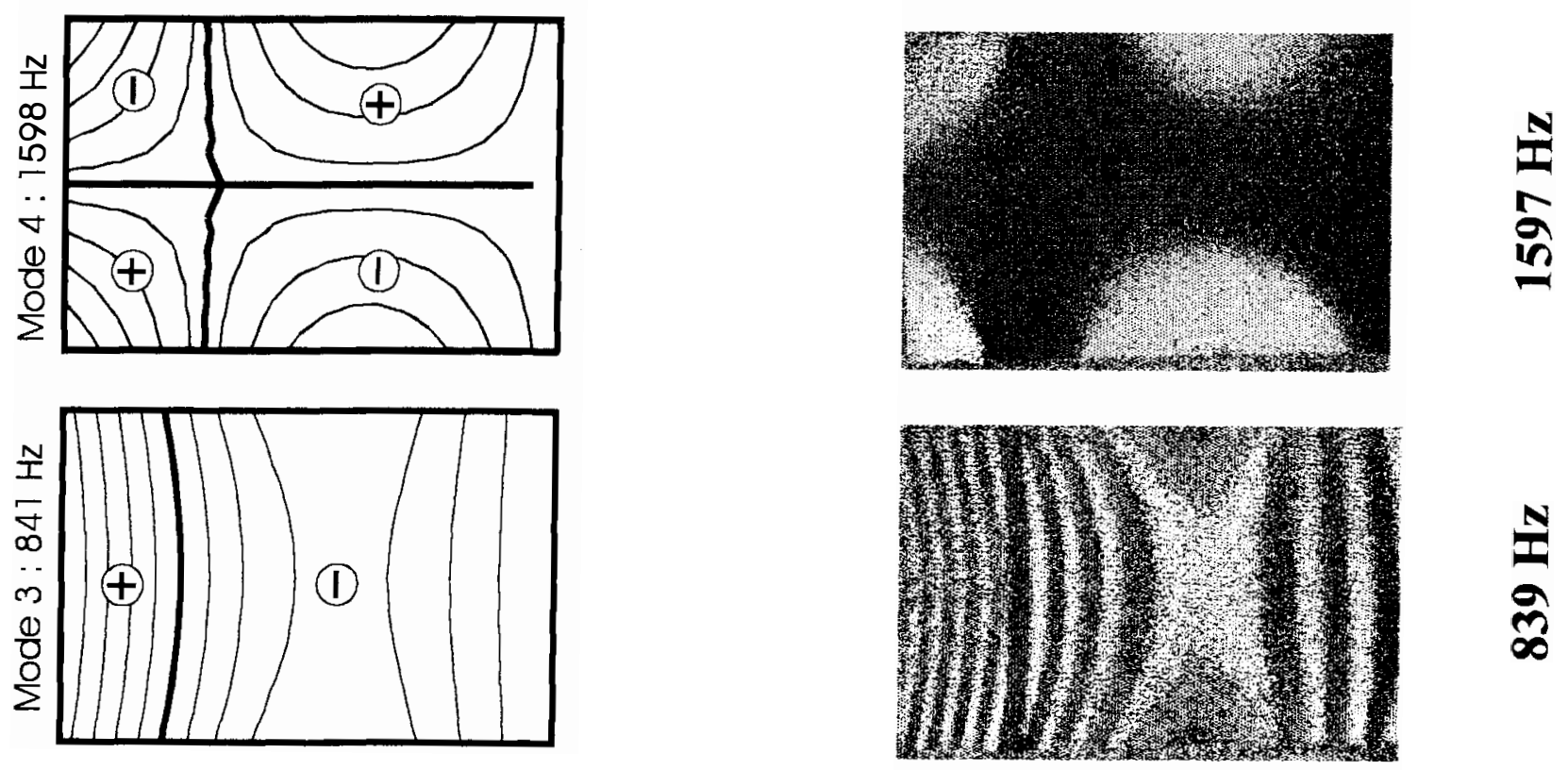

m
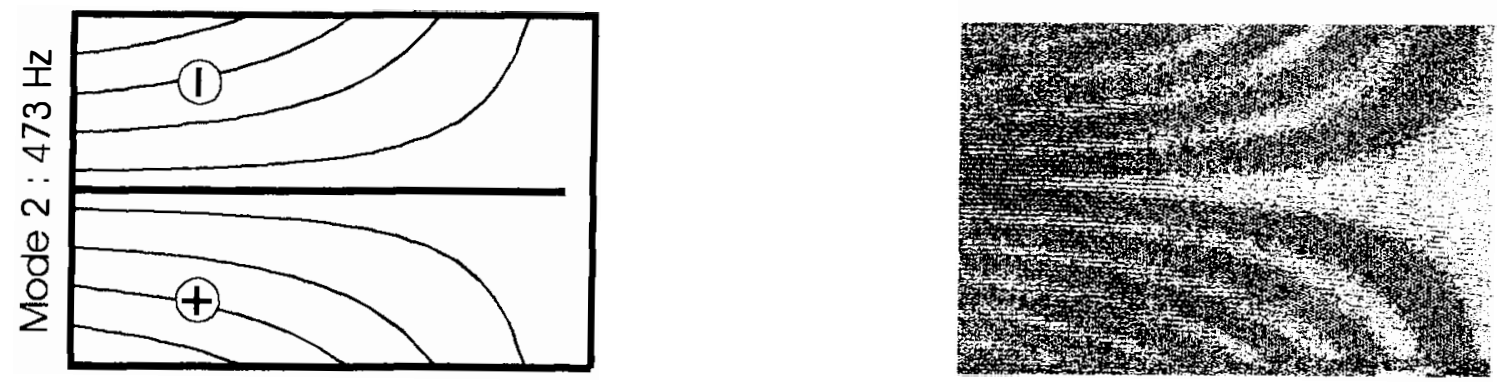

$\stackrel{N}{\stackrel{0}{*}}$
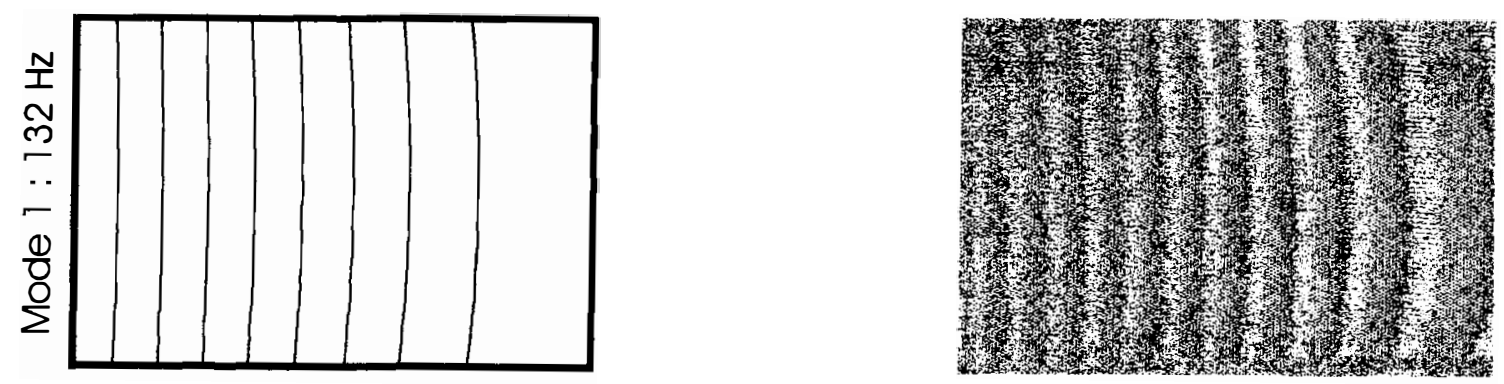

$\frac{N}{m}$

Fig. 9 Comparison of computed (FEM) and DSPI modes 


\section{REFERENCES}

1. R. JONES, C. WYKES, Holographic and speckle interferometry, second edition, Cambridge university press, 1989.

2. F. MENDOZA SANTOYO, M. C. SHELLABEAR, J. R. TYRER, "Whole field in-plane vibration analysis using pulsed phase-stepped ESPI », Appl. Opt., Vol.30(7), pp.717-721, 1991.

3. L.EK, N.E. MOLIN, « Detection of the nodal lines and the amplitude of vibration by speckle interferometry », Optics Comm., Vol.2 (9), pp.419-424, 1971.

4. O. J. LOKBERG, K. HOGMOEN, "Vibration phase mapping using electronic speckle pattern interferometry ", Appl. Opt., Vol.15(11), pp.2701-2704, 1976.

5. K. HOGMOEN, O. J. LOKBERG, « Detection and measurement of small vibrations using electronic speckle pattern interferometry », Appl. Opt., Vol.16(7), pp.1869-1875, 1977.

6. J. C. DAVIES, J. D. C. JONES, «Developments in ESPI for automotive vibration analysis », Proc.SPIE, Vol.952, pp.260-269, 1988.

7. K. HOGMOEN, H. M. PEDERSEN, «Measurement of small vibrations using electronic speckle pattern interferometry : Theory », J.Opt.Soc.Am. A, Vol.67(11), pp.1578-1583, 1977.

8. K. CREATH, G. A. SLETTEMOEN, «Vibration-observation techniques for digital speckle-pattern inferferometry ", J.Opt.Soc.Am. A, Vol.2 (10), pp.1629-1636, 1985.

9. P. C. MONTGOMERY, B. D. BERGQUIST, « Contrast enhancement of ESPI vibration patterns by speckle averaging in a video frame store ", Proc.SPIE, Vol.599, pp201-206, 1985.

10. I. A. POPOV, L. M. VESELOV, «Vibration analysis by means of a speckle method», Optik, Vol.92(3), pp.119-122, 1993.

11. K. CREATH, « Phase-shifting speckle interferometry », Appl. Opt., Vol.24 (18), pp.3053-3058, 1985.

12. C. JOENATHAN, "Vibration fringes by phase stepping on an electronic speckle pattern interferometer : an analysis », Appl. Opt., Vol.30(32), pp.4658-4665, 1991.

13. E. VIKHAGEN, "Vibration measurement using phase shifting TV-Holography and Digital Image Processing ", Optics Comm., 1989, Vol.69 (3,4), pp.214-218

14. G. JIN, S. TANG, « Electronic speckle pattern interferometer with a polarization phase-shift technique », Opt.Eng., Vol.31(4), pp.857-860, April 1992.

15. L. HOLDER, T. OKAMOTO, T.ASAKURA, « Polarization phase-shifting technique applied to a speckle correlation interferometer using an image fiber », Optik, Vol.96, pp.120, 1994.

16. C. R. MERCER, G. BEHEIM, «Speckle Interferometry using fiber optic phae stepping», Laser interferometry, Vol.11 62, pp.180-186, 1989.

17. SAMCEF, Asef, Dynam, Repdyn User Manual, Samtech, Revision 5.0, Liège, 1992.

18. R.D. Blevins, Formulas for Natural Frequency and Mode Shape, Van Nostrand Reinhold, 1979.

19. A. R GANESAN, P. MEINLSCHMIDT, K. D. HINSCH, "Vibration mode separation using comparative electronic speckle pattern interferometry (ESPI) », Optics Comm., Vol.107, pp.28, 1994.

20. M. C. SHELLABEAR, J. R. TYRER, « Three-dimensional vibration analysis using ESPI », Proc.SPIE, Vol.952, pp.251-259, 1988. 